\title{
GRIPSTRENGTH, HAND FUNCTION AND DAILY ACTIVITIES IN PATIENTS WITH SYSTEMIC LUPUS ERYTHEMATOSUS WITH VERSUS WITHOUT JACCOUD'S ARTHROPATHY: CROSS-SECTIONAL STUDY
}

Verena Loureiro Galvão ${ }^{1,2, \star}$, Mittermayer B. Santiago $0^{1,3,4}$

1.Escola Bahiana de Medicina e Saúde Pública, Salvador (SP), Brazil. 2.Universidade Católica do Salvador, Salvador (BA), Brazil. 3.Serviços Especializados em Reumatologia da Bahia, Salvador (BA), Brazil. 4.Hospital Universitário Professor Edgard Santos, Salvador (BA), Brazil.

*Corresponding author: verenaloureiro@gmail.com

\section{BACKGROUND}

Joint involvement is a major clinical manifestation of systemic lupus erythematosus (SLE). Jaccoud's arthropathy (JA) is a "reducible" deforming arthritis that can occur in up to 5\% of SLE patients. This study aimed to assess and compare grip strength, hand function and the degree of functional disability among patients with SLE, with or without JA.

\section{MATERIALS AND METHODS}

A cross-sectional study was conducted among patients with SLE, with or without JA, paired by age. Patients' selection was done using convenience sampling. The disability index of Health Assessment Questionnaire (HAQDI) and HandHAQ, a modified version of the HAQ and Michigan Health Outcomes Questionnaire (MHQ) were used. These questionnaires were self-completed by patients to avoid suggestions for completion. Bilateral grip strength was also assessed, being considered the highest value. Study sample size was calculated to be 27 patients.

\section{RESULTS}

Overall, 56 female patients with SLE (28 with JA) were included. The average age of patients with JA and SLE was $47.79( \pm 12.18)$ years and that of the SLE without JA group was $45.46( \pm 12.91)$ years. Most frequent articular deformities found in SLE with JA group were "swan neck" and "ulnar deviation" (20 patients, $71.42 \%$ ). The HAQDI scores had no statistically significant differences in various domains between groups, with exception of domain "reach", which showed a borderline significance $(p=0.051)$. With regard to the final HAQDI score, a median of $1.12(0.78-1.31)$ was observed in the SLE with JA group and $1.0(0.66-1.25)$ in the SLE without JA group, indicating moderate difficulties in the performance of daily activities and the need for special assistance. Hand Health Assessment Questionnaire and $\mathrm{MHQ}$ domains revealed no statistically significant differences between the groups. The grip strength of the right hand was $0(0.0-4.0) \mathrm{KgF}$ and that of the left hand was $0(0.0-6.0) \mathrm{kgF}$ in the SLE with JA group, while that in the SLE without JA group was $5(1.25-13.75) \mathrm{kgF}(p=0.001)$ and $7.5(4.25-17.25) \mathrm{KgF}(p=0.001)$, respectively.

\section{CONCLUSION}

Hand function and daily activities of SLE patients with and without JA, present dysfunction and reduced performance related to the daily activities, but with no statistical difference between groups; however, the grip strength was reduced bilaterally in SLE patients with JA. 